\title{
Do we know all about Behavioral and Psychological Symptoms of Dementia?
}

\section{Aleš Kogoj ${ }^{1 *}$ and Tanja Prokšelj}

${ }^{1}$ Psychogeriatric Department, University Psychiatric Hospital, Ljubljana, Slovenia ${ }^{2}$ General Hospital Novo mesto, Neuropsychiatric Department, Novo mesto, Slovenia

With the aging of population, dementia is becoming an increasingly prevalent mental disorder. Dementia is associated with progressive cognitive disability and a high prevalence of behavioral and psychological symptoms (BPSD) sometimes also named non-cognitive symptoms. Nearly all patients with dementia experience at least one behavioral or psychological symptom during the disease progress [1]. BPSD also contribute to increased stress and decreased quality of life for patients and their caregivers [2].

The International Psychogeriatric Association Consensus group recognized that for certain purposes it might be useful to group different psychiatric and behavioral symptoms into specific symptom clusters: behavioral symptoms (usually identified on the basis of observation of the patient, including physical aggression, screaming, restlessness, agitation, wandering, culturally inappropriate behaviors, sexual disinhibition, hoarding, cursing and shadowing) and psychological symptoms (usually and mainly assessed on the basis of interviews with patients and relatives; these symptoms include anxiety, depressive mood, hallucinations and delusions) [3]. Other authors recognized also numerous other symptoms: apathy, wandering, pacing, rummaging, alterations in sleep and appetite, and involuntary emotional expression disorder $[4,5]$.

The neurobiology of BPSD is still unclear. Several neurotransmitter systems are involved in Alzheimer's disease and other dementias in addition to decline of cholinergic system. They may precede cholinergic decline as they play role in different affective and psychotic disorders. Abnormal functioning of the serotonergic system is well proved in depressed mood and anxiety, but also in agitation, restlessness, and aggressiveness. Beside that dopaminergic system plays a role in cognitive function it may be in addition related with psychosis and apathy. An imbalance between the glutamate and dopaminergic systems may also result in psychotic symptoms. A significant decrease in cholinergic activity results not only in memory decline but also in hypomanic or manic symptoms, delusions, hallucinations and physical aggression [6,7].

In addition to non-pharmacological interventions, use of various medications offers the best chance to alleviate BPSD, reduce family burden and lower societal costs in patients with dementia. BPSD generally respond better to therapy than other symptoms or syndromes of dementia [3].

However, there are some obstacles for successful treatment of BPSD. Appropriate recognizing and assessing BPSD is not always easy due to cognitive decline and ineffective communication with patient with dementia. Sometimes one can rely only on observation which can also be misleading. Side-effects of antipsychotics can be mistaken with psychosis, weak sight or misidentifications can be erroneously interpreted as hallucinations, and confabulations as delusions. In addition, depression, for example, often presents with atypical symptoms which can easily be overlooked.

Some symptoms like alterations in sleep and appetite or agitation can occur due to several causes, each one requiring different approach. Agitation can for example occur not only due to cognitive decline (when effective communication is not possible, or due to inappropriate orientation), depression, psychosis, delirium or sundowning syndrome, but also due to pain, somatic diseases (dehydration, urinary retention, infections etc.), loss of sight or hearing, medication, noise and social isolation. The relevant cause must be identified in order that appropriate social, environmental, behavioral or medical intervention can be made to alleviate the symptom.

A clinician faced with BPSD cannot turn a blind eye to the patient and its family. Therefore many drugs are used to alleviate BPSD, even if they are used off-label. Unfortunately, some drugs were found to cause unexpected side-effects in patients with dementia. Antipsychotics, for example, which are also often used for treatment of psychotic symptoms and severe aggressive behavior in dementia are known for an increased risk of cerebrovascular events in those patients [8].

To improve quality of life of patients with dementia and their caregivers better treatment of BPSD with less side-effects is needed. One of symptoms not fully understood is involuntary emotional expression disorder (IEED), also called pseudobulbar affect. IEED is a syndrome characterized by involuntary episodes of crying or laughing that typically occur suddenly and appear to be independent or in excess of any eliciting stimulus or the prevailing mood. The development of this syndrome was associated with serotonin (5-hydroxytryptamine, 5-HT) [9], dopamine [10], glutamate and sigma-1 receptors [11].

Currently the only drug approved for treating pseudobulbar affect is dextromethorphan, uncompetitive NMDA ionotropic glutamate receptor antagonist, in combination with quinidine which is a potent CYP2D6 inhibitor thus influencing metabolism of several other drugs. Studies to support the effectiveness of dextromethorphan/quinidine were performed in patients with amyotrophic lateral sclerosis and multiple sclerosis, but not in other types of emotional lability that can commonly occur, for example, in Alzheimer's disease and other dementias. The question remains whether neurobiology of IEED in Alzheimer's disease is the same as in neurological diseases.

The recent study showed that Alzheimer's disease patients with IEED have also significantly lower platelet 5-HT concentrations compared to patients with aggressive behavior or patients without BPSD. As this study also pointed out on a small number of patients, the use of a selective 5-HT reuptake inhibitor (SSRI) decreased number of episodes of involuntary emotional expression [12]. However, more research has to be done, before it could be recommended for treatment of IEED.

While effective causal treatment remains elusive for even the most

*Corresponding author: Aleš Kogoj, MD, Associated Professor of Psychiatry Psychogeriatric Department, University Psychiatric Hospital, Studenec 48, SI-1260 Ljubljana Polje, Slovenia, Tel: 38641412178; E-mail: ales.kogoj@mf.uni-lj.si

Received November 14, 2013; Accepted November 16, 2013; Published November 16, 2013

Citation: Kogoj A, Prokšelj T (2013) Do we know all about Behavioral and Psychological Symptoms of Dementia? J Gerontol Geriat Res 3: e124. doi:10.4172/2167-7182.1000e124

Copyright: $\odot 2013$ Kogoj A, et al. This is an open-access article distributed under the terms of the Creative Commons Attribution License, which permits unrestricted use, distribution, and reproduction in any medium, provided the original author and source are credited. 
Citation: Kogoj A, Prokšelj T (2013) Do we know all about Behavioral and Psychological Symptoms of Dementia? J Gerontol Geriat Res 3: e124. doi:10.4172/2167-7182.1000e124

frequent causes of dementia (Alzheimer's disease, dementia with Lewy bodies, frontotemporal dementias), understanding underlying mechanisms of BPSD could help us to choose the most appropriate drug.

\section{References}

1. Youn JC, Lee DY, Jhoo JH, Kim KW, Choo IH, et al. (2011) Prevalence of neuropsychiatric syndromes in Alzheimer's disease (AD). Arch Gerontol Geriatr 52: 258-263.

2. Allegri RF, Sarasola D, Serrano CM, Taragano FE, Arizaga RL, et al. (2006) Neuropsychiatric symptoms as a predictor of caregiver burden in Alzheimer's disease. Neuropsychiatr Dis Treat 2: 105-110.

3. International Psychogeriatric Association (2002) Behavioral and Psychological Symptoms of Dementia (BPSD) Educational Pack. International Psychogeratric Association, Belgium.

4. Mega MS, Cummings JL, Fiorello T, Gornbein J (1996) The spectrum of behavioral changes in Alzheimer's disease. Neurology 46: 130-135.

5. Cummings JL, Arciniegas DB, Brooks BR, Herndon RM, Lauterbach EC, et al. (2006) Defining and diagnosing involuntary emotional expression disorder. CNS Spectr 11: 1-7.
6. Reeves SJ, Gould RL, Powell JF, Howard RJ (2012) Origins of delusions in Alzheimer's disease. Neurosci Biobehav Rev 36: 2274-2287.

7. Cerejeira J, Lagarto L, Mukaetova-Ladinska EB (2012) Behavioral and psychological symptoms of dementia. Front Neurol 3: 73

8. Finkel S, Kozma C, Long S, Greenspan A, Mahmoud R, et al. (2005) Risperidone treatment in elderly patients with dementia: relative risk of cerebrovascular events versus other antipsychotics. Int Psychogeriatr 17: 617-629.

9. Arciniegas DB, Topkoff $J(2000)$ The neuropsychiatry of pathologic affect: an approach to evaluation and treatment. Semin Clin Neuropsychiatry 5: 290-306.

10. Lauterbach EC, Schweri MM (1991) Amelioration of pseudobulbar affect by fluoxetine: possible alteration of dopamine-related pathophysiology by selective serotonin reuptake inhibitor. J Clin Psychopharmacol 11: 392-393.

11. Panitch HS, Thisted RA, Smith RA, Wynn DR, Wymer JP, et al. (2006) Randomized, controlled trial of dextromethorphan/quinidine for pseudobulbar affect in multiple sclerosis. Ann Neurol 59: 780-787.

12. Prokšelj T, Jerin A, Kogoj A (2012) Memantine may affect pseudobulbar affect in patients with Alzheimer disease. Acta Neuropsychiatrica. 